\title{
NONREGULAR CONTACT STRUCTURES ON BRIESKORN MANIFOLDS ${ }^{1}$
}

\author{
BY KINETSU ABE AND JOSEPH ERBACHER
}

Communicated by Shlomo Sternberg, December 2, 1974

1. Introduction. In 1958, Boothby and Wang [1] showed that if a compact, $(2 n-1)$-dimensional differentiable manifold $M^{2 n-1}$ admits a regular contact structure, then $M^{2 n-1}$ is the total space of a principal $S^{1}$ bundle over a symplectic manifold. To the authors' knowledge no examples of nonregular contact structures on compact manifolds have appeared in the literature. The purpose of this announcement is to exhibit a large class of compact manifolds that admit a contact structure; this class of manifolds includes the Brieskorn and generalized Brieskorn manifolds, and, in particular, those exotic spheres that arise as Brieskorn manifolds. Furthermore, when $n=2$, these contact structures are often nonregular.

In the next section we recall the relevant definitions, state our main theorems, and indicate their proofs. The details will appear elsewhere.

2. Let $M^{2 n-1}$ be a $(2 n-1)$-dimensional differentiable manifold. A contact structure on $M^{2 n-1}$ is a 1 -form $\omega$ that satisfies

$$
\omega \wedge(d \omega)^{n-1} \neq 0 \text { everywhere. }
$$

A distribution $V$ is associated with $\omega$ as follows. Let

$$
V_{p}=\left\{X \in T_{p}\left(M^{2 n-1}\right) \mid d \omega(X, Y)=0 \forall Y \in T_{p}\left(M^{2 n-1}\right)\right\} .
$$

Because of (1), $\operatorname{dim} V_{p}=1$. Thus, $V$ is integrable and determines a one-dimensional foliation of $M^{2 n-1}$. The contact structure is called regular if this foliation is regular in the sense of foliations [6] ; otherwise, it is called nonregular. Recall that a foliation is called regular if for each $p \in M^{2 n-1}$ there exists Froebenius coordinates around $p$ such that different slices belong to different leaves.

AMS (MOS) subject classifications (1970). Primary 53 C15.

${ }^{1}$ This research was partially supported by NSF Grant GP 43980 . 
Our main theorems are the following.

THEOREM 1. Let $N$ be a complex submanifold of $C^{n+1}$. Let $S^{2 n+1}$ be a standard sphere in $C^{n+1}$. Suppose that $N$ intersects $S^{2 n+1}$ transversally in a differentiable manifold $M\left(=N \cap S^{2 n+1}\right)$. Then the restriction of the standard contact form on $S^{2 n+1}$ to $M$ is a contact form on $M$.

The contact form above is the same contact form considered indepenently by Hsu and Sasaki [3] when $M$ is a Brieskorn manifold.

For example, let $N$ be an algebraic variety minus its singular points defined by a single polynomial with the origin as an isolated singular point or a regular point. Let $S^{2 n+1}$ be a sphere of small radius $\epsilon$ with center at the origin. For $\epsilon$ sufficiently small, it is well known [4] that $N$ intersects $S^{2 n+1}$ transversally. If the polynomial is a Brieskorn polynomial [2], [4] or weighted homogenous, we obtain the Brieskorn or generalized Brieskorn manifolds, which includes those exotic spheres which bound parallelizable manifolds. Incidentally, the above transversality holds for any complex algebraic set in $C^{n+1}$ near an isolated singular point or a regular point.

LEMMA. If $M^{3}$ is a compact three-dimensional manifold with a regular contact form, then either $\pi_{1}\left(M^{3}\right)$ contains an isomorphic copy of $Z$ or $\pi_{1}\left(M^{3}\right)$ is cyclic.

\section{THEOREM 2. There exists infinitely many three-dimensional compact} manifolds with a nonregular contact structure.

OUtLINE OF PROOFS. Let us assume that the sphere $S^{2 n+1}$ has its center at the origin of $C^{n+1}$. The standard contact form $\widetilde{\omega}$ on $S^{2 n+1}$ is given by $\widetilde{\omega}(X)=\langle X, J \vec{x}\rangle$, where $\vec{x}$ is the position vector in $C^{n+1}, J$ the complex structure on $C^{n+1}$, and $\langle$,$\rangle is the standard inner product on C^{n+1}$. Let $\omega$ be the restriction of $\widetilde{\omega}$ to $M$. The proof of Theorem 1 depends upon the fact that transversality implies that the tangent space $T_{p}(M)$ at each $p \in$ $M$ is spanned by a complex subspace of $T_{p}\left(C^{n+1}\right)$ and the projection of $J \vec{x}(p)$ onto $T_{p}(M)$. Using this and the intrinsic definitions of the exterior derivative and the exterior product, it is not difficult to show that $\omega \wedge(d \omega)^{k}$ $\neq 0$, where $2 k+1=\operatorname{dim} M$. One simply evaluates $\omega \wedge(d \omega)^{k}$ on an orthonormal basis $X_{1}, X_{2}, \ldots, X_{2 k+1}$, where $X_{1}, \ldots, X_{2 k}$ spans the complex subspace of $T_{p}(M)$.

The lemma is an easy consequence of the Boothby-Wang fibration theorem [1] and the homotopy exact sequence of a fibration. 
Theorem 2 is proved by noting that the generalized Brieskorn manifold associated to the polynomial $z_{1}^{2}+z_{2}^{2} z_{3}+z_{3}^{k+1}$ has fundamental group equal to the binary dihedral group with $4 k$ elements [4], [5]; and this group is a finite, noncommutative group. Therefore, by the lemma, the contact structure guaranteed by Theorem 1 is nonregular.

The other examples known to the authors of compact manifolds with a nonregular contact structure are the Brieskorn and generalized Brieskorn manifolds with fundamental groups the binary icosohedral group, the binary tetrahedral group, the binary octahedral group, and the quaternion group [4].

ADDED IN PROOF.

REMARK 1. It has been pointed out to the authors that S. Tanno, Sasakian manifolds with constant $\phi$-holomorphic sectional curvature, Tohoku Math. J. 21 (1969), 501-507 has shown that certain $(4 n+3)$-dimensional compact manifolds of constant positive curvature admit a nonregular contact structure.

REMARK 2. Recently, Montgomery and Yang, Differentiable actions on homotopy seven spheres. II, Proceedings of the Conference on transformation groups, New Orleans, 1967, Springer-Verlag, New York (1968), pp. 125-134 showed that there are certain exotic 7-spheres which do not admit any free $S^{\mathbf{1}}$-action. Combining that result with the Boothby-Wang fibration theorem, the contact structures exhibited above are nonregular on those exotic spheres.

\section{BIBLIOGRAPHY}

1. W. M. Boothby and H. C. Wang, On contact manifolds, Ann. of Math. (2) 68 (1958), 721-734. MR 22 \#3015.

2. E. V. Brieskorn, Beispiele zur Differentialtopologie von Singularïäten, Invent. Math. 2 (1966), 1-14. MR 34 \#6788.

3. C. J. Hsu and S. Sasaki, On a property of Brieskorn manifolds (preprint).

4. J. W. Milnor, Singular points of complex hypersurfaces, Ann. of Math. Studies, no. 61, Princeton Univ. Press, Princeton, N. J., Univ. of Tokyo Press, Tokyo, 1968. MR 39 \#969.

5. P. Orlik, Weighted homogenous polynomials and fundamental groups, Topology 9 (1970), 267-273. MR 41 \#6251.

6. R. S. Palais, A global formulation of the Lie theory of transformation groups, Mem. Amer. Math. Soc. No. 22 (1957). MR 22 \#12162.

DEPARTMENT OF MATHEMATICS, UNIVERSITY OF CONNECTICUT, STORRS, CONNECTICUT 06268

DEPARTMENT OF MATHEMATICS, UNIVERSITY OF SOUTHERN CALIFORNIA, LOS ANGELES, CALIFORNIA 90007 